\title{
Personality Disorders and Their Relation to Treatment Outcomes in Cognitive Behavioural Therapy for Depression: A Systematic Review and Meta-analysis
}

\author{
Harry Banyard ${ }^{1} \cdot$ Alex J. Behn ${ }^{2,3} \cdot$ Jaime Delgadillo ${ }^{4}$
}

Accepted: 5 January 2021 / Published online: 20 January 2021

(c) The Author(s) 2021

\begin{abstract}
Background Previous reviews indicate that depressed patients with a comorbid personality disorder (PD) tend to benefit less from psychotherapies for depression and thus personality pathology needs to be the primary focus of treatment. This review specifically focused on studies of Cognitive Behavioural Therapy (CBT) for depression examining the influence of comorbid PD on post-treatment depression outcomes.

Methods This was a systematic review and meta-analysis of studies identified through PubMed, PsychINFO, Web of Science, and Scopus. A review protocol was pre-registered in the PROSPERO database (CRD42019128590).

Results Eleven eligible studies $(N=769)$ were included in a narrative synthesis, and ten $(N=690)$ provided sufficient data for inclusion in random effects meta-analysis. All studies were rated as having "low" or "moderate" risk of bias and there was no significant evidence of publication bias. A small pooled effect size indicated that patients with PD had marginally higher depression severity after CBT compared to patients without PD ( $\mathrm{g}=0.26$, [95\% CI: 0.10, 0.43], $p=.002)$, but the effect was not significant in controlled trials $(p=.075)$, studies with low risk of bias $(p=.107)$ and studies that adjusted for intake severity $(p=.827)$. Furthermore, PD cases showed symptomatic improvements across studies, particularly those with longer treatment durations (16-20 sessions).

Conclusions The apparent effect of PD on depression outcomes is likely explained by higher intake severity rather than treatment resistance. Excluding these patients from evidence-based care for depression is unjustified, and adequately lengthy CBT should be routinely offered.
\end{abstract}

Keywords Cognitive behavioural therapy $\cdot$ Depression $\cdot$ Personality disorder $\cdot$ Systematic review $\cdot$ Meta-analysis

\section{Introduction}

Supplementary Information The online version contains supplementary material available at https://doi.org/10.1007/s1060 8-021-10203-x

Jaime Delgadillo

jaime.delgadillo@nhs.net

1 Department of Psychology, University of Sheffield, Sheffield, UK

2 Department of Psychology, Pontificia Universidad Católica de Chile, Santiago, Chile

3 Millennium Institute for Research in Depression and Personality (MIDAP), Santiago, Chile

4 Clinical Psychology Unit, Department of Psychology, University of Sheffield, Floor F, Cathedral Court, 1 Vicar Lane, Sheffield S1 2LT, UK
Personality disorders (PD) refer to an enduring pattern of inner experiences and interpersonal difficulties that significantly deviate from the individual's culture, which are inflexible and pervasive over a range of social scenarios. This pattern can often be traced back to adolescence or early adulthood and is not better explained as a manifestation of another mental health problem (American Psychiatric Association 2013). According to a recent systematic review (Winsper et al. 2019), the pooled global prevalence of PD has been estimated to be around $7.8 \%$, with higher prevalence rates found in high-income countries $(\sim 9.6 \%)$ compared with low and middle-income countries $(\sim 4.3 \%)$. Cluster $C$ disorders, characterized by fearfulness and anxiety (avoidant, dependent, and obsessive-compulsive) are the most common in the general population with an estimated prevalence 
of $5.0 \%$. This is followed by Cluster A disorders with a prevalence of $3.8 \%$, characterized by eccentric beliefs and interpersonal styles (paranoid, schizoid, and schizotypal). Cluster $B$ disorders have the lowest estimated prevalence around $2.8 \%$, and are characterized by intense emotional lability (antisocial, borderline, histrionic, and narcissistic).

PD are associated with considerable psychological distress, social problems, functional impairment, and premature mortality (Moran et al. 2016). Clinical guidelines for the management of PD recommend psychotherapy as a first line treatment (Simonsen et al. 2019). In spite of this, individuals with a PD are less likely to seek treatment compared to those with other mental health conditions (Andrews et al. 2001), and particularly those with Cluster A disorders are more likely to reject treatment (Tyrer et al. 2003). Previous studies indicate that PD are highly comorbid with each other (Ekselius et al. 1994; McGlashan et al. 2000) and with other mental health problems (McGlashan et al. 2000). It is likely that frequent comorbidity among personality disorders is an artifact of an imperfect polythetic diagnostic system that assumes the existence of different and discrete types of PDs. This system has been substantially modified more recently, among other reasons because it produces high rates of comorbidity, dropping the different types of categorical PDs in favour of one single diagnosis of PD estimated within a severity continuum (Herpertz et al. 2017). In regards to comorbidity with other mental health problems, PDs are particularly comorbid with major depressive disorder (Corruble et al. 1996). For example, between 40 and $80 \%$ of depressed patients have been estimated to have comorbid PD, depending on the treatment setting, with cluster $\mathrm{C}$ disorders being most common (Friborg et al. 2014; Hirschfeld 1999; Wongpakaran et al. 2015).

Although the comorbidity of depression and PD is widely documented, the impact of personality pathology on depression treatment outcomes has been a matter of controversy for many years. Earlier systematic reviews on this topic reported mixed and inconclusive evidence (Kool et al. 2005; Mulder 2002). Conversely, more recent reviews indicate that poorer treatment outcomes were found for depressed patients diagnosed with comorbid personality disorders (Newton-Howes et al. 2006, 2014). The latest of these reviews (NewtonHowes et al. 2014) reported this overall finding consistently across subgroup analyses pooling data from electroconvulsive therapy samples, pharmacotherapy samples, psychotherapy samples and combined treatments. However, the specificity of this effect is less clear, since the meta-analysis for psychotherapy studies combined data from different forms of treatment (e.g., cognitive behavioural therapy, counselling, psychodynamic therapy, and other unspecified therapies). This is problematic because it has been suggested that, for example, specific types of psychotherapy may differentially benefit patients with depression and avoidant versus obsessive personality traits (Barber and Muenz 1996), although this evidence is still inconclusive (Kikkert et al. 2016). Moreover, relatively few studies of cognitive behavioural therapy (CBT) were included in this review, despite the fact that CBT is one of the most widely studied and recommended treatments for depression (Chambless and Ollendick 2001; National Institute for Health and Care Excellence 2011). Other systematic reviews examining psychotherapy outcomes in the context of PD have tended to have a narrow scope, for example focusing on borderline personality disorder (e.g., Cristea et al. 2017; Davidson and Tran 2014) which has a comparatively low prevalence in typical clinical samples with depression (Wongpakaran et al. 2015).

Newer studies have emerged on the relation between PD and depression treatment outcomes (e.g., Harte and Hawkins 2016; van Bronswijk et al. 2018), warranting a more focussed review of the evidence pertinent to contemporary CBT practice, and specifically of the effectiveness of CBT for depression in patients with comorbid PDs. This has potentially profound clinical implications. A co-morbid PD is found in roughly half of the patients who present with depression across clinical services and is predictive of suboptimal clinical outcomes for depression. As a result, clinical algorithms developed for the care of patients presenting this comorbidity have typically posed that personality pathology needs to be addressed first, using evidence-based PD treatments, before depressive symptomatology can be improved (Gunderson 2014; Van and Kool 2018). This becomes problematic from the perspective of treatment availability and delivery, since evidence-based treatments for PDs are underdeveloped for disorders other than borderline personality disorder and in general very costly in time and money (Spong et al. 2020) and largely unavailable in health care systems across the globe (Paris 2020). Compared to evidence-based care for PDs, psychotherapy for depression is more often available across health care systems, particularly in middle and high income countries, less costly and time consuming (Araya et al. 2018). CBT is one of the most widely studied and recommended treatments for depression (Chambless and Ollendick 2001; National Institute for Health and Care Excellence 2011) and, to the extent that it may be effective for the treatment of depression comorbid with PD, this would result in a large expansion of the availability of care for this group of patients when the primary treatment target is depressive symptomatology. Furthermore, this would lead to a potential modification of commonly used clinical algorithms and clinical wisdom regarding treatment sequences for patients with PDs (i.e., personality pathology needs to be treated before, in order to improve depressive symptomatology).

On this basis, the present study aimed to fill this gap in the literature through a systematic review and quantitative synthesis of CBT-specific studies investigating associations 
between personality disorders and depression treatment outcomes.

\section{Methods}

The review protocol was registered in the international prospective register of systematic reviews (PROSPERO) database prior to the search being conducted (registration number: CRD42019128590). Informed consent from study participants was not required, as this study was a secondary analysis of results from primary studies that are available in scientific journals.

\section{Search Strategy}

The inclusion and exclusion criteria described in Table 1 guided the development of a systematic search strategy which combined key terms (related to personality disorder, CBT, depression) using Boolean operators. The search was limited to peer-reviewed articles published in the English language, and was applied on 29th March 2019 across four databases: PsychINFO, SCOPUS, Web of Science, and PubMed. No restrictions were imposed on study publication dates. Furthermore, in order to derive findings that are treatment-specific, we only included studies that applied bona fide CBT treatment protocols, excluding related variations or adaptations (mindfulness-based interventions; ACT; DBT; CBASP; guided self-help adaptations of CBT) or components of CBT (i.e., behavioural activation). The full search strategy can be found in the online supplement (Online Appendix A).

Titles and abstracts were screened by the first author and relevant information was collated using the Cochrane Collaboration data extraction form (Higgins et al. 2019). Reverse-citations and reference list searches were conducted by hand on eligible studies to identify any further relevant studies.

\section{Primary Outcome}

The primary outcome of interest was depression treatment outcome, as determined by diagnostic interview or using validated self-rated or clinician-rated depression symptom measures. This review specifically focused on studies where depression treatment outcomes were compared between participants with and without a personality disorder diagnosis.

\section{Risk of Bias Assessment}

Risk of bias was assessed using the CASP tool for cohort studies (Critical Appraisal Skills Programme 2018) and the revised Cochrane risk of bias tool for randomised trials
(Sterne et al. 2019). Eligible papers were assessed independently by two reviewers who later compared their ratings and arrived at a consensus without the need for moderation by a third reviewer. The reviewers had no disagreements in their final risk of bias ratings, so an interrater reliability calculation was deemed redundant and is therefore not reported.

\section{Data Synthesis}

A narrative (qualitative) review of all reviewed studies was conducted. A random effects meta-analysis was additionally completed for all studies that provided sufficient statistical information, using the statistical $\mathrm{R}$ package Meta-Analysis via Shiny (MAVIS; Hamilton et al. 2016). Statistical information examining between-group (No PD vs. PD) differences was standardised using Hedges' $g$ to enable meta-analysis, with adjustment for unequal sample sizes across groups, and to correct for small-sample bias. A single within-study pooled effect size was calculated for studies that reported more than one measure of depression symptoms. Where available, effect sizes adjusted for intake depression severity were selected for meta-analysis. Q and $\mathrm{I}^{2}$ statistics enabled the examination of heterogeneity (Higgins et al. 2003). A weight function model and fail-safe $N$ calculation using Rosenthal's method were also employed to investigate publication bias (Orwin 1983). The relatively small number of studies included in meta-analysis $(<20)$ precluded the use of more detailed moderator analyses (Rubio-Aparicio et al. 2017), so we limited our investigation of heterogeneity using subgroup analyses according to study design features (experimental vs. observational; depression outcome measures; PD diagnosis methods; risk of bias ratings; adjustment for intake depression severity). Finally, an exploratory analysis examined non-parametric (Spearman's) correlations between effect sizes and the maximum duration of the CBT protocol used across studies (since mean treatment duration was not consistently reported).

\section{Results}

\section{Sample Characteristics and Measures}

A PRISMA diagram summarising the search and selection process is displayed in Fig. 1. Eleven studies met the inclusion criteria and are described in Tables 2 and 3. The majority were randomised controlled trials $(\mathrm{k}=7)$ and the remainder were observational cohort studies $(k=4)$. As shown in Table 3, the specific number of CBT patients with depression across all included studies was 769 (sample size range $=24-122)$. More than half $(64.6 \%)$ of participants across studies were females, mostly of a White Caucasian background (65-89\%), and the mean age across 


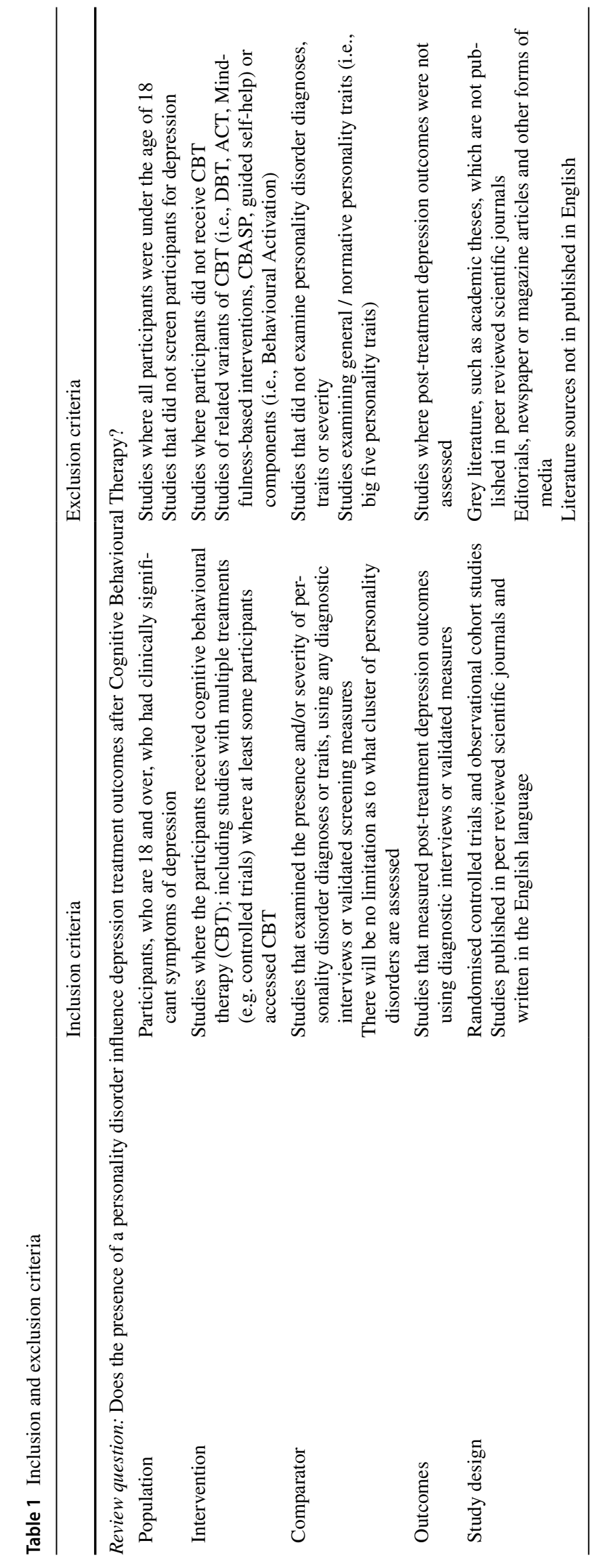




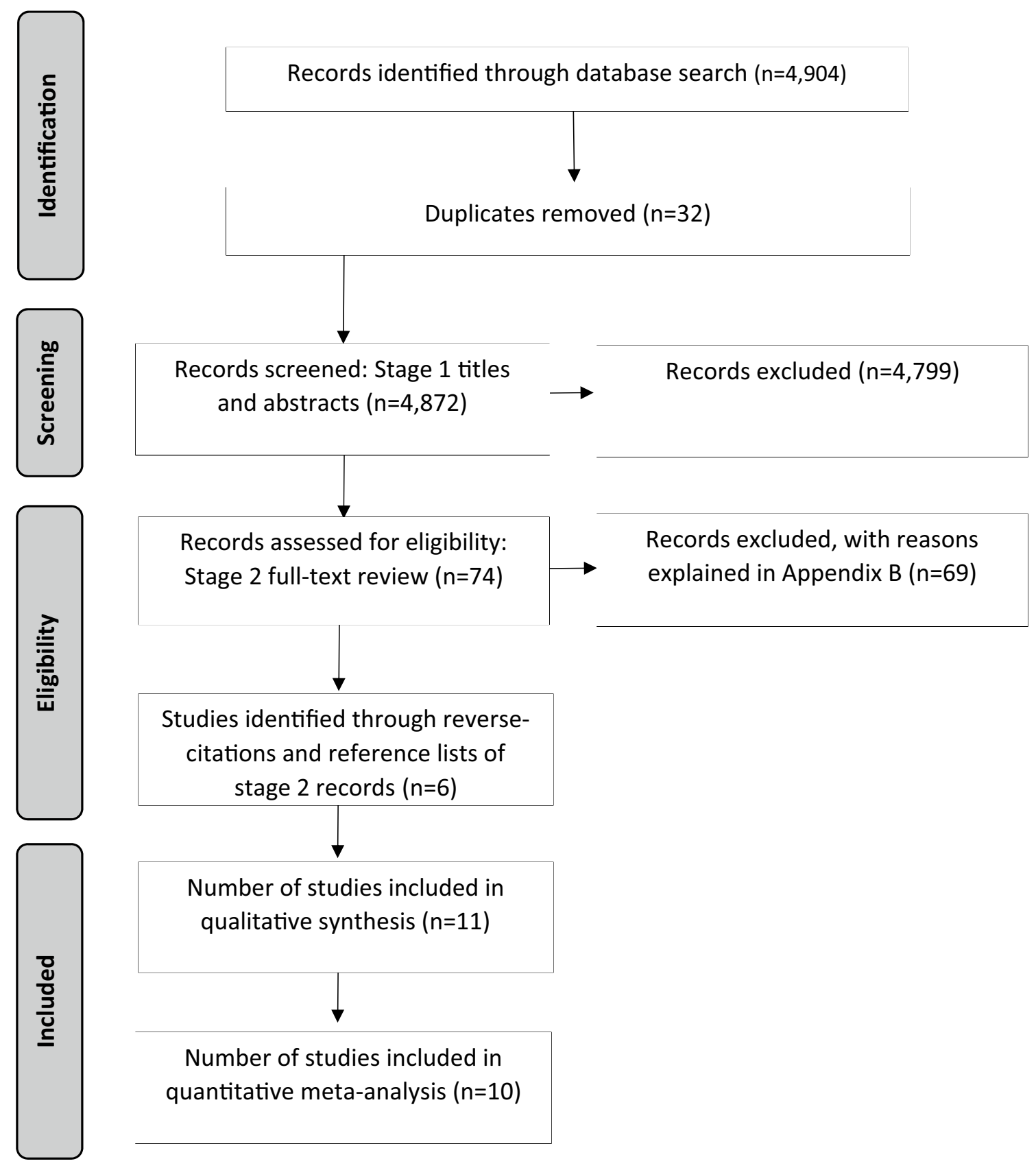

Fig. 1 PRISMA flow diagram of the systematic study selection

samples ranged from 32 to 42.5 . All studies were conducted in western countries. Employment status ranged considerably across studies (30-91\%).

Most studies used self-reported depression measures as the primary outcome as shown in Table 2. The Beck Depression Inventory (BDI, BDI-II) and Hamilton Rating Scale for Depression (HRSD) were the two most commonly used measures. Other measures included the Montgomery-Åsberg Depression Rating Scale (MADRS) and the Hopkins Symptom Checklist 90-item major depressive disorder subscale (HSCL-90 MDD).

Personality disorder diagnoses across most studies were obtained using the Structured Clinical Interview for the DSM $(\mathrm{k}=7)$. A variety of editions of the DSM were used: DSM-III $(n=1)$, DSM-III-R $(n=5)$, DSM-IV $(\mathrm{k}=2)$. Craigie et al. (2007), used the Millon Clinical Multiaxial Inventory-III to classify an individual's personality disorder complexity. van den Hout et al. (2006) conducted two 


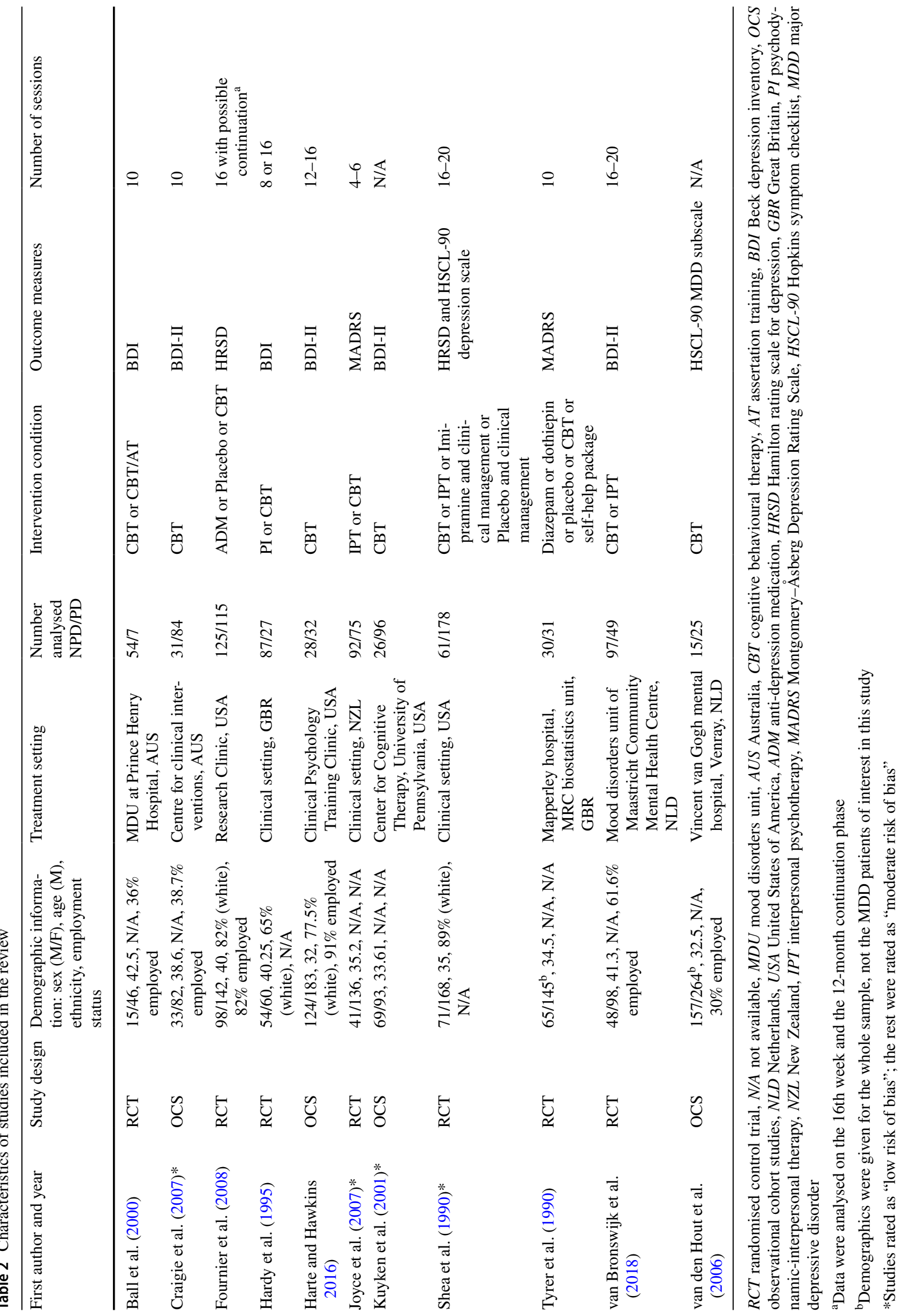


Table 3 Main findings reported by studies included in the review

First author and year $\quad$ Personality disorder complex- Personality disorder type $(N)$ CBT sample sizes ity $(N)$

Ball et al. (2000)

N/A

Cluster A: 0

Cluster B: 14

Cluster C: 40

PD NOS: 0

Craigie et al. (2007)

Simple PD: 50

Complex PD: 34

No PD: 31

Cluster A: 38

Cluster B: 24

Cluster C: 81

PD NOS: 76

Fournier et al. (2008)

N/A

Cluster A: 8

Cluster B: 8

Cluster C: 74

PD NOS: 35

Hardy et al. (1995)

Simple PD: 18

Complex PD: 9

No PD: 87

Cluster A: 0

Cluster B: 0

Cluster C: 27

PD NOS: 0
$\mathrm{NPD}=33$

Summary of key findings specific to CBT samples

PD cases had higher mean depression severity at intake NPD cases had greater improvements in depression symptoms posttreatment and at long-term follow-up (BDI post-treatment: $\mathrm{NPD}=9.0$ vs. $\mathrm{PD}=19.55$; pooled $\mathrm{SD}=13.25)$. There were no significant differences between PD Clusters over the assessment periods ( $F=1.4$, NS)

Results were not adjusted for intake depression severity

$\mathrm{NPD}=31$

$\mathrm{PD}=84$

PD cases had higher mean depression severity at intake

Chi-square analysis showed that the NPD group had a significantly higher frequency of CSI compared to the simple PD group, $\chi^{2}$ (1, $N=79)=8.29, p<.01$, and the complex PD group, $x^{2}$ (1, $N=63)=4.37, p<05$

BDI posttreatment: $\mathrm{NPD}=12.52(\mathrm{SD}=9.79)$; $\mathrm{PD}=19.55(\mathrm{SD}=25.6)$

Results were not adjusted for intake depression severity

Differences in intake depression severity in NPD vs. PD cases were not reported

Depression treatment response rates were $\mathrm{NPD}=70 \%$ vs. $\mathrm{PD}=44 \%$ within the cognitive therapy group

Results within the CT group were not adjusted for intake depression severity

PD cases had higher mean depression severity at intake

No significant differences in depression treatment outcome were found comparing PD and NPD groups; $F(1,45)=1.10, p=0.30$

Results were adjusted for intake depression severity 
Table 3 (continued)

First author and year

Harte and Hawkins 2016)
Personality disorder complex- Personality disorder type $(N)$ CBT sample sizes ity $(N)$
Summary of key findings specific to CBT samples

Cluster A: 4

Cluster B: 10

Cluster C: 18

PD NOS: 5

Joyce et al. (2007)

Simple PD: 52

Complex PD: 23

No PD/dysfunction: 92

Cluster A: 26

Cluster B: 20

Cluster C: 56

PD NOS: 0

Cluster A: 3

Cluster B: 23

Cluster C: 40

PD NOS: 30
$\mathrm{NPD}=13$

$\mathrm{PD}=11$

Intake depression severity scores were highly similar between NPD and PD cases

Among patients treated for depression, similar proportions of patients in the NPD (40.6\%) and PD (39.3\%) groups recovered after treatment; adjusted $\mathrm{OR}=1.07$ (95\% CI 0.34, 3.39), $p=\mathrm{NS}$

Results were adjusted for intake depression severity

$\mathrm{NPD}=37$

$\mathrm{PD}=43$

Differences in intake depression severity in NPD vs. PD cases were not reported

PD did not significantly predict post-treatment outcome for patients treated with CBT $\left(\mathrm{R}^{2}=0.018, p=\mathrm{NS}\right)$. Posttreatment \% improvement in MADRS score: $\mathrm{NPD}=66.1$ $(\mathrm{SD}=33.8) ; \mathrm{PD}=57.5$ $(\mathrm{SD}=28.7)$

Results were not adjusted for intake depression severity

PD cases had higher mean depression severity at intake

The comparison of outcome by personality disorder was not significant for either self-rated depression severity or clinical-rated global functioning

BDI posttreatment: $\mathrm{NPD}=7.23$ $(\mathrm{SD}=10.55) ; \mathrm{PD}=9.54$ $(\mathrm{SD}=12.57)$

Results were not adjusted for intake depression severity 
Table 3 (continued)

First author and year Personalit ity $(N)$

Shea et al. (1990)

Simple PD: 76

Complex PD: 102

No PD: 78

Tyrer et al. (1990)

van Bronswijk et al (2018) Simple PD: 37 Complex PD: 12 No PD: 97
Simple PD: 35

Complex PD: 37

No PD: 87
Cluster B: 40

Cluster C: 155

PD NOS: 0

Cluster A: 22

Cluster B: 43

Cluster C: 25

PD NOS: 0

Cluster A: 5

Cluster B: 3

Cluster C: 39

PD NOS: 2
Summary of key findings specific to CBT samples

Differences in intake depression severity in NPD vs. PD cases were not reported

Within the CBT treatment condition NPD cases had similar albeit marginally higher depression severity compared to PD cases; this pattern was consistent in clinicianreported and patient-reported measures

HRSD posttreatment:

$\mathrm{NPD}=11.94(\mathrm{SD}=7.45)$;

$\mathrm{PD}=10.34(\mathrm{SD}=7.45)$

SCL-90 depression scale posttreatment: NPD $=1.30$ $(\mathrm{SD}=0.97) ; \mathrm{PD}=1.23$ $(\mathrm{SD}=1.01)$

Pooled effect size across both measures, using Hedge's g estimation: $\mathrm{g}=-0.14$

Results were adjusted for intake depression severity

PD cases had higher mean depression severity at intake

In the full sample including pharmacological, self-help and CBT treatments, PD cases had significantly higher posttreatment impairment across all measures, even after adjusting for intake severity. NPD and PD cases in the CBT treatment arm had similar levels of improvement over time

Results were adjusted for intake depression severity

PD cases had higher mean depression severity at intake

Within the CBT treatment arm, post-treatment BDI scores were highly similar between NPD and PD cases, albeit marginally higher in the NPD group

BDI posttreatment: NPD $=13.6$ $(\mathrm{SD}=9.4) ; \mathrm{PD}=13.3$ $(\mathrm{SD}=11.3)$

Results were not adjusted for intake depression severity (in the specific contrast of NPD vs. PD within the CBT arm) 
Table 3 (continued)

First author and year Personality disorder complex- Personality disorder type $(N)$ CBT sample sizes ity $(N)$

van den Hout et al. (2006) N/A

Cluster A: 0

Cluster B: 1

Cluster C: 5

PD NOS: 19

Totals ( within studies that Total $=957$

reported each feature) Simple PD: $268(28.0 \%)$

Complex PD: 217 (22.7\%)

No PD: $472(49.3 \%)$

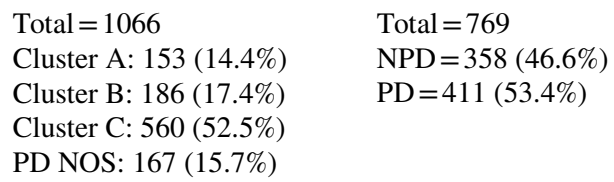

PD NOS: 167 (15.7\%)

\author{
Total $=769$ \\ $\mathrm{NPD}=358(46.6 \%)$ \\ $\mathrm{PD}=411(53.4 \%)$
}

Summary of key findings specific to CBT samples

PD cases had higher mean depression severity at intake Within the group of depressed CBT patients, the proportions with clinically significant improvement were $\mathrm{NPD}=80 \%$ vs. $\mathrm{PD}=52 \%\left(\chi^{2}\right.$ $[\mathrm{df}=1]=3.1, p=0.038$ )

Results were not adjusted for intake depression severity

The prevalence of personality disorder clusters is taken from the largest available sample in each study, in order to minimise selection bias and
to render more accurate estimates of overall prevalence in typical clinical samples. The column of statistical results is drawn specifically from
subsamples of CBT cases in studies where the full sample may have included other treatments (i.e., pharmacotherapy or other psychological interventions)

N/A not available, $P D$ personality disorder, NOS not otherwise specified, NPD no personality disorder

Cluster A refers to: paranoid, schizoid, and schizotypal personality disorders

Cluster B refers to: antisocial, borderline, histrionic, and narcissistic personality disorders

Cluster C refers to: avoidant, dependent, and obsessive-compulsive personality disorders

PD NOS (personality disorders not otherwise specified) refers to problems with inter and intrapersonal functioning that are not accounted for by the specific diagnoses defined by the Diagnostic Statistical Manual (DSM) or International Classification of Diseases (ICD)

clinical interviews with each of their patients but did not specify their diagnostic criteria. Shea et al. (1990) did not conduct clinical interviews but used a "Personality Assessment Form" which screened for PD features based on the DSM-III. Tyrer et al. (1990) used a "Personality Assessment Schedule" as their clinical interview guide.

\section{Prevalence of Personality Disorders}

As shown in Table 3, most studies $(\mathrm{k}=10)$ assessed different personality clusters in their sample, with the most common being cluster C (52.5\%) across all studies. Six studies assessed personality disorder complexity, where "simple PD" refers to a single PD diagnosis and "complex PD" refers to cases meeting criteria for two or more PD. Pooled data from available studies indicates that approximately $49.3 \%$ of study participants did not have a PD, $28.0 \%$ met criteria for a single PD, and $22.7 \%$ met criteria for two or more PD (complex PD). Furthermore, CBT-specific samples across included studies indicated that around half $(53.4 \%)$ of patients met criteria for a PD.

\section{Risk of Bias Assessment}

Most studies generally had low risk of bias. Five studies were rated as "low risk" and six studies were rated as "moderate risk". No studies were regarded as "high risk". The main sources of bias were lack of inclusion of all PD clusters, not analysing missing outcomes data, unclear randomisation procedures and blinding in clinical trials, and not addressing the implications of the outcomes found in the study. Tables showing risk of bias ratings for all reviewed studies can be found in the online supplement.

\section{Narrative Synthesis of Findings Across Studies}

Key findings in CBT-specific samples are presented in Table 3. Most studies $(k=7)$ reported higher intake depression severity scores for cases with PD; three studies did not report intake severity comparisons (Fournier et al. 2008; Joyce et al. 2007; Shea et al. 1990) and only one study reported similar intake severity scores (Harte and Hawkins II). PD cases were also more likely to have a higher number of comorbid Axis I disorders and overall clinical and social impairment at intake. However, few studies $(\mathrm{k}=4)$ analysed treatment outcome comparisons adjusted for intake depression severity (Hardy et al. 1995; Harte \& Hawkins II; Shea et al. 1990; Tyrer et al. 1990).

Across most studies $(\mathrm{k}=9)$ PD cases tended to have higher mean depression symptoms after treatment, although the magnitude and significance of this trend varied considerably across studies (see Table 3). Only two studies reported 
Fig. 2 Random effects metaanalysis: effect of personality depression severity disorder (PD) on post-treatment

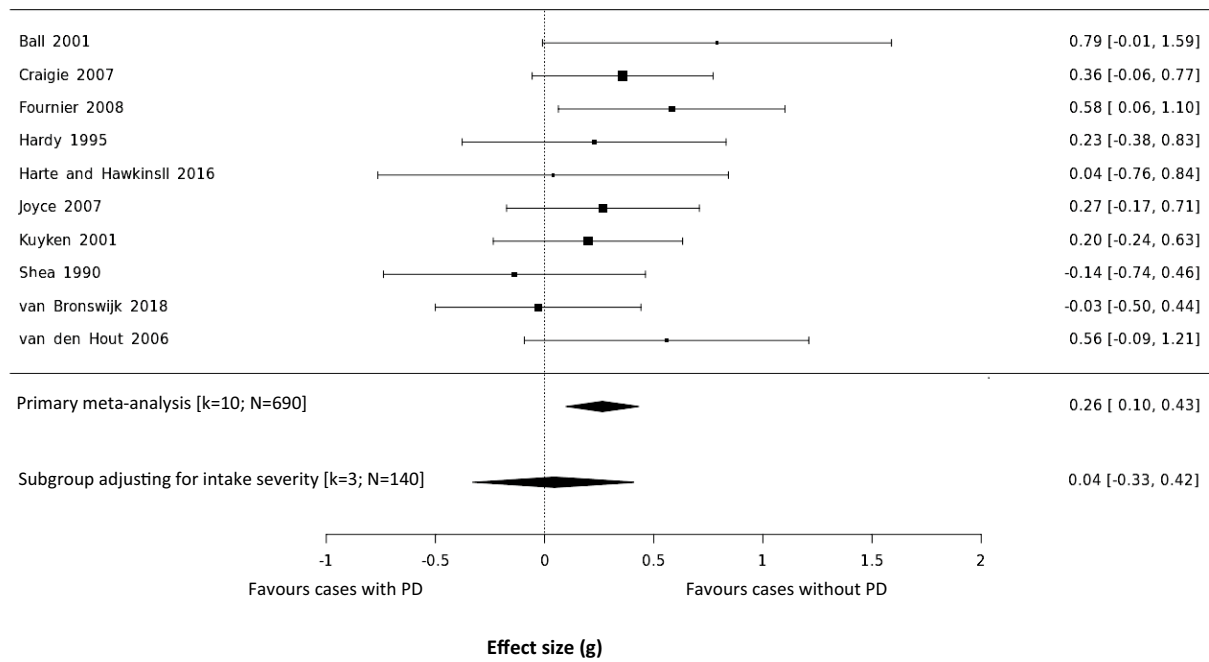

the opposite trend, where cases with no PD had marginally higher depression severity after treatment, but this was not statistically significant (Shea et al. 1990; van Bronswijk et al. 2018). Although some studies indicated that PD cases tended to improve less than those without PD, PD cases did nevertheless benefit from CBT and often made considerable improvements. For example, Hardy et al. (1995) reported a large within-group effect size $(d=2.41)$ on the BDI measure for CBT cases with a PD, albeit in a small sample $(N=14)$. A study with a larger sample $(N=54)$ also showed a large $(d=1.18)^{1}$ within-group effect size on the BDI measure for PD cases treated with CBT (Ball et al. 2000). Furthermore, improvements in depression severity were maintained for cases with PD in studies that carried out longer-term followup assessments (e.g., Ball et al. 2000; Hardy et al. 1995; van Bronswijk et al. 2018).

Studies examining the relationship between PD complexity and treatment outcomes had mixed and contradictory findings. Similarly, studies that examined the relationship between PD and dropout/attrition had inconsistent findings. Joyce et al. (2007) and Kuyken et al. (2001) examined relationships between specific personality traits and posttreatment BDI-II scores. Joyce et al. (2007) did not find significant associations between specific PD diagnoses and CBT treatment outcomes. The study by Kuyken et al. (2001) found that, although PD diagnosis was unrelated to CBT treatment outcomes, maladaptive avoidant and paranoid beliefs predicted poorer treatment outcomes after controlling for intake severity (avoidance predicted poorer depression; paranoia predicted poorer general functioning).

\footnotetext{
1 This effect size was calculated pooling available pre- and post-treatment means and standard deviations for Cluster B and Cluster $\mathrm{C}$ cases with PD.
}

\section{Meta-analysis}

Ten studies $(N=690)$ provided sufficient data for inclusion in the primary meta-analysis examining associations between PD and post-treatment depression severity. The weighted mean effect size was $\mathrm{g}=0.26$, (95\% CI $0.10,0.43$ ), $p=0.002$, indicating that the presence of a PD was significantly associated with higher post-treatment depression severity (see Fig. 2). Cochran's Q-test $(Q[9]=7.71, p=0.56$ ) and the $\mathrm{I}^{2}$ statistic $(0.0 \%)$ indicated no significant evidence of heterogeneity. The regression for funnel plot asymmetry and Kendall's tau indicated no significant evidence of publication bias, $t(8)=0.47, p=0.652$; Kendall's $\tau=0.07$, $p=0.862$; Failsafe $N=26$. The corresponding funnel plot for the main analysis is available in the online supplement (Online Appendix D).

Sub-group analyses were conducted to examine the potential influence of methodological features on the magnitude of the observed effect size. The effect size in the subgroup of studies using the BDI depression measure $(\mathrm{k}=6$; $N=451)$ was similar to the main analysis $(\mathrm{g}=0.23$, [95\% CI $0.01,0.44], p=0.037)$ and with no significant heterogeneity $\left(Q[5]=3.65, p=0.601, \mathrm{I}^{2}=0.0 \%\right)$. The effect size in the subgroup of studies using DSM-based diagnostic interviews for $\mathrm{PD}(\mathrm{k}=7 ; N=476)$ was similar to the main analysis $(\mathrm{g}=0.26$, [95\% CI 0.06, 0.46], $p=0.012)$ and with no significant heterogeneity $\left(Q[6]=5.00, p=0.544, \mathrm{I}^{2}=0.0 \%\right)$. The effect size in the subgroup of studies rated as having low risk of bias $(\mathrm{k}=5 ; N=448)$ was smaller compared to the main analysis $(\mathrm{g}=0.17,[95 \% \mathrm{CI}-0.04,0.38])$, not statistically significant $(p=0.107)$, and with no significant heterogeneity $\left(Q[4]=2.69, p=0.610, \mathrm{I}^{2}=0.0 \%\right)$. The effect size in the subgroup of randomised controlled trials $(\mathrm{k}=6 ; N=389)$ was similar to the main analysis $(\mathrm{g}=0.25,[95 \% \mathrm{CI}-0.03$, $0.52])$, but not statistically significant $(p=0.075)$, and 
with no significant heterogeneity $(Q[5]=6.29, p=0.279$, $\mathrm{I}^{2}=31.5 \%$ ). The effect size in the subgroup of studies that adjusted for intake depression severity $(\mathrm{k}=3 ; N=140)$ was small and non-significant $(\mathrm{g}=0.04$, [95\% CI $-0.33,0.42$ ], $p=0.827$ ), compared to the results from studies that did not adjust for intake severity $(\mathrm{k}=7 ; N=550 ; \mathrm{g}=0.32,[95 \%$ CI $0.13,0.51], p<0.001)$. Tests for heterogeneity in both of these subgroup analyses were not significant $(p>0.05$; $\left.\mathrm{I}^{2}=0.0 \%\right)$.

A strong and statistically significant inverse correlation was found between CBT treatment-protocol duration and effect sizes $(r=-0.76, p=0.016)$.

\section{Discussion}

\section{Summary of the Evidence}

This systematic review and meta-analysis found that patients meeting diagnostic criteria for a PD tended to improve less than patients without a PD after CBT for depression. There was no significant evidence of publication bias and the failsafe $N$ calculation suggested that 26 studies with null findings would be required to contradict the primary meta-analysis results. However, the effect size for this association was small $(g=0.26)$ and was not statistically significant in samples from RCTs, studies with low risk of bias, and studies that adjusted for intake depression severity. According to our narrative synthesis of the literature, there was some evidence of depression intake severity differences between cases with simple vs. complex PD, but little evidence that PD complexity influenced treatment outcomes, although there was insufficient data across studies to verify this observation using a moderator analysis. Furthermore, the apparent detrimental effect of PD was lower in studies that offered longer CBT treatments, as evidenced by a strong $(r=-0.76)$ and significant negative correlation between effect sizes and treatment duration. Finally, there was mixed and inconclusive evidence of associations between PD with treatment dropout.

\section{Methodological and Conceptual Issues}

There was considerable variability in methods used to assess PD and depression across all included studies. However, we found no significant evidence of effect size heterogeneity related to diagnostic interviews or depression rating tools. The main methodological sources of variability in outcomes were related to study design (RCTs vs. observational studies), risk of bias, and adjustment for intake severity.

Most studies reported higher intake severity and impairment in PD cases. Prior evidence suggests that PDs are associated with adverse early life events (e.g., Porter et al. 2020), with an earlier onset of comorbid Axis I disorders such as depression (Corruble et al. 1996) and thus greater severity and dysfunction by the time that patients access treatment. Despite this evident relationship between PD status and initial impairment, few studies $(\mathrm{k}=4)$ analysed treatment outcome comparisons adjusted for intake depression severity, and associations between PD and treatment outcomes in this subgroup were minimal in magnitude $(\mathrm{g}=0.04)$ and not statistically significant. This suggests that the apparently adverse effect of PD is mostly explained by intake severity rather than some form of treatment resistance or intractability. This explanation is further supported by the observation that PD cases did in fact improve across all studies, and treatment gains were maintained at longer follow-ups. The effect of PD was not significant in RCTs, which is plausibly explained by the extent to which CBT was closely supervised and adherence closely monitored, particularly in studies with low risk of bias-which also showed non-significant effects of PD. Taken together, these findings suggest that patients with PD tend to be more severely depressed and impaired, but they can benefit considerably from CBT in contexts where therapy is closely supervised, delivered with adherence to treatment manuals, and with a sufficiently lengthy treatment duration (e.g., 16-20 sessions). This conclusion fits with prior meta-analytic studies that indicate that the efficacy of CBT is well-established in the context of personality disorders (e.g., see Hofmann et al. 2012).

Basic conceptual issues regarding diagnosis of depression and personality disorders should also be considered. First, depression is increasingly viewed as a rather heterogeneous clinical entity (Fried and Nesse 2015). Thus, research looking at the concurrent presentation of depression and PD may not be nuanced enough to draw relevant and conclusive findings. For example, Köhling et al. (2015) isolated a specific phenotype of depression in patients diagnosed with Borderline Personality Disorder (BPD), and in an important longitudinal study over 24 years, Zanarini et al. (2019) discovered that depression was significantly more likely to be present in patients with BPD compared to patients with other PD, even though recurrence and remission rates over time were comparable in both groups. It is possible that CBT may be particularly suited for cases in which a true comorbidity is present, but limited in cases where depression is secondary to PD. These diagnostic nuances may partially dilute overall effects when depression is viewed as a consistent syndrome. If they are, in fact, distinct disorders, psychotherapies including CBT should exhibit a good effect on depressive symptomatology regardless of comorbid personality dysfunction. If the opposite is true and both disorders are related, then psychotherapy may exhibit restricted effectiveness. This may be the case for depression in the context of BPD, or in depressive phenotypes characterized by excessive self-criticism as proposed by Huprich (2019) and Dinger et al. (2015). Furthermore, the temporal stability 
of PD diagnosis varies across PD subtypes (e.g., 63\% for cluster B and $48 \%$ for cluster C).

\section{Strengths and Limitations}

To our knowledge, this review is the first to specifically assess the influence of PD on depression treatment outcomes after CBT. The study shows several hallmarks of good practice in systematic review; such as the pre-registration of the study protocol ahead of conducting searches, a comprehensive and inclusive search strategy applied across multiple databases, forward and reverse citation searches, a doublerated risk of bias assessment, and a quantitative synthesis using meta-analysis.

There are, however, also some limitations that warrant consideration. In spite of its inclusivity of studies without a publication date restriction, the review excluded any form of grey literature and studies that were not written in the English language. Whilst subgroup analyses were conducted to investigate methodological sources of heterogeneity, the relatively small set of studies precluded more robust moderator analyses. Only five studies reported attrition rates and assessed whether there was any relationship between dropout and PD diagnosis. Even though none of these studies found a significant relationship between dropout and PD diagnosis, this does not rule out the potential influence of attrition bias in the remaining studies. Similarly, only six studies reported PD complexity, so the findings in relation to complexity are derived from a small subset. An additional limitation of the study concerns the issue of severity in personality disorders. Most studies included in this review used DSM-IV categorical diagnoses without the inclusion of a dimensional severity gradient, which would enable a better examination of the relevance of PD severity. New studies that measure personality dysfunction on a dimensional scale and which control for pre-treatment depression severity could enable future moderator analyses. Finally, the variable follow-up durations across the few studies that examined longer-term effects precluded an examination of long-term treatment outcomes and relapse rates in relation to PD.

\section{Implications for Research and Practice}

Previous reviews by Newton-Howes et al. $(2006,2014)$ are indicative of a detrimental effect of PD in psychotherapy studies, with a small-to-moderate effect size $(\mathrm{OR}=2.01$; approximate $d=0.38$ ). On this basis, clinical guidelines and conventional wisdom often suggest that when depression and PDs are concurrent, PD needs to be the main therapeutic target. However, as they state, their review is broad and it looks at all treatment types, both psychopharmacology and psychotherapy, whereas the present study specifically focuses on CBT. In the current meta-analysis, the overall effect of
PD on depression outcomes after CBT is smaller $(g=0.26)$ than previously assumed, and not statistically significant in contexts where CBT is delivered with high fidelity (i.e., in controlled trials) and with a longer treatment duration. Even though CBT delivery in most real-world clinical settings is less controlled than in clinical trials, this evidence suggests that depression can be improved in patients with concurrent depression and PDs, and that traditional sequencing algorithms could in fact be modified, resulting in an expansion of access to evidence-based treatment for this group of patients.

An important theoretical question arising from this literature concerns how to optimize CBT for the treatment of depressed patients with a PD. One possibility is that allowing for a higher-than-average treatment duration may in itself be sufficient to allow for severe symptomology (typical of PD samples) to subside. Another possibility is that incorporating treatment strategies specifically targeting core beliefs (e.g., Beck et al. 2015) may be necessary to help patients with more chronic and entrenched maladaptive beliefs and interpersonal difficulties. For example, the study by Kuyken et al. (2001) found that PD diagnostic status was unrelated to depression treatment outcomes, but avoidant and paranoid maladaptive beliefs predicted poorer treatment response even after adjusting for intake severity. Future studies could empirically test these hypotheses, for example by randomising depressed patients with PD to low intensity (i.e., eight sessions) CBT without core-belief work versus high-intensity CBT (i.e., 16-20 sessions) with versus without core belief work. Further research is also needed to explore which specific traits and domains of personality dysfunction account for a restriction of therapeutic benefits of psychotherapy in general and CBT in particular, and which components of $\mathrm{PD}$ are innocuous in this regard, or even beneficial, as in the case of anaclitic depressive styles (Luyten et al. 2017).

The diagnosis of PD has experienced significant shifts in the last few years, particularly since the publication of ICD-11 where different types of PD are dropped in favour of a single diagnosis of Personality Disorder and a gradient of severity (Herpertz et al. 2017). Yet, it remains controversial in mental healthcare, and it has been argued that it can result in stigma and exclusion from evidence-based treatments (Kealy and Ogrodniczuk 2010; Snowden and Kane 2003; Stalker et al. 2005). When clinicians wish to help their patients with their depressive symptoms, in our view, the modest effect of PD on depression treatment outcomes can hardly justify the widespread clinical algorithm of postponing evidence-based psychotherapy for depression in favour of treatments targeting PDs and thus, CBT should be routinely available to them, particularly in the setting of stepped-care models (Paris 2013; Spong et al. 2020). Still, the routine assessment of specific PD traits (and related maladaptive beliefs and behaviours) could support the 
personalised delivery of CBT to make it more responsive to the needs of this patient group. The adoption of reliable screening of personality dysfunction in routine CBT practice could improve therapists' ability to personalise treatment, by including the individual's personality traits and relational history in case formulation and treatment planning.

Acknowledgements We would like to thank Adam McCombe for his assistance with independent risk of bias ratings of included studies.

Funding No external funding.

\section{Compliance with Ethical Standards}

Conflict of Interest Harry Banyard, Alex J. Behn and Jaime Delgadillo declare that they have no conflict of interest.

Ethical Approval This review article was exempt from ethical review because it did not collect any primary research data from human participants.

Animal Rights No animal studies were carried out by the authors for this article.

Open Access This article is licensed under a Creative Commons Attribution 4.0 International License, which permits use, sharing, adaptation, distribution and reproduction in any medium or format, as long as you give appropriate credit to the original author(s) and the source, provide a link to the Creative Commons licence, and indicate if changes were made. The images or other third party material in this article are included in the article's Creative Commons licence, unless indicated otherwise in a credit line to the material. If material is not included in the article's Creative Commons licence and your intended use is not permitted by statutory regulation or exceeds the permitted use, you will need to obtain permission directly from the copyright holder. To view a copy of this licence, visit http://creativecommons.org/licenses/by/4.0/.

\section{References}

American Psychiatric Association. (2013). Diagnostic and statistical manual of mental disorders (5th ed.). Arlington, VA: APA.

Andrews, G., Issakidis, C., \& Carter, G. (2001). Shortfall in mental health service utilisation. The British Journal of Psychiatry, 179, 417-425. https://doi.org/10.1192/bjp.179.5.417.

Araya, R., Zitko, P., Markkula, N., Rai, D., \& Jones, K. (2018). Determinants of access to health care for depression in 49 countries: A multilevel analysis. Journal of Affective Disorders, 234, 80-88. https://doi.org/10.1016/j.jad.2018.02.092.

Ball, J., Kearney, B., Wilhelm, K., Dewhurst-Savellis, J., \& Barton, B. (2000). Cognitive behaviour therapy and assertion training groups for patients with depression and comorbid personality disorders. Behavioural and Cognitive Psychotherapy, 28, 71-85. https://doi. org/10.1017/S1352465800000072.

Barber, J. P., \& Muenz, L. R. (1996). The role of avoidance and obsessiveness in matching patients to cognitive and interpersonal psychotherapy: Empirical findings from the treatment for depression collaborative research program. Journal of Consulting and Clinical Psychology, 64, 951-958. https://doi. org/10.1037/0022-006X.64.5.951.
Beck, A. T., Davis, D. D., \& Freeman, A. (2015). Cognitive therapy of personality disorders. New York: Guilford Publications.

Critical Appraisal Skills Programme (2018). CASP systematic review checklist. [online]. Retrieved March 20, 2019, from https://caspuk.net/wp-content/uploads/2018/01/CASP-Economic-Evaluation -Checklist-2018.pdf.

Chambless, D. L., \& Ollendick, T. H. (2001). Empirically supported psychological interventions: Controversies and evidence. Annual Review of Psychology, 52, 685-716. https://doi.org/10.1146/annur ev.psych.52.1.685.

Corruble, E., Ginestet, D., \& Guelfi, J. D. (1996). Comorbidity of personality disorders and unipolar major depression: A review. Journal of Affective Disorders, 37(2-3), 157-170. https://doi. org/10.1016/0165-0327(95)00091-7.

Craigie, M. A., Saulsman, L. M., \& Lampard, A. M. (2007). MCMI-III Personality complexity and depression treatment outcome following group-based cognitve-behavioral therapy. Journal of Clinical Psychology, 63, 1153-1170. https://doi.org/10.1002/jclp.20406.

Cristea, I. A., Gentili, C., Cotet, C. D., Palomba, D., Barbui, C., \& Cuijpers, P. (2017). Efficacy of psychotherapies for borderline personality disorder: A systematic review and meta-analysis. JAMA Psychiatry, 74(4), 319-328. https://doi.org/10.1001/jamapsychi atry.2016.4287.

Davidson, K. M., \& Tran, C. F. (2014). Impact of treatment intensity on suicidal behavior and depression in borderline personality disorder: A critical review. Journal of Personality Disorders, 28(2), 181-197. https://doi.org/10.1521/pedi_2013_27_113.

Dinger, U., Barrett, M. S., Zimmermann, J., Schauenburg, H., Wright, A. G., Renner, F., \& Barber, J. P. (2015). Interpersonal problems, dependency, and self-criticism in major depressive disorder. Journal of Clinical Psychology, 71, 93-104. https://doi.org/10.1002/ jclp. 22120.

Ekselius, L., Lindström, E., von Knorring, L., Bodlund, O., \& Kullgren, G. (1994). Comorbidity among the personality disorders in DSM-III-R. Personality and Individual Differences, 17(2), 155-160. https://doi.org/10.1016/0191-8869(94)90021-3.

Friborg, O., Martinsen, E. W., Martinussen, M., Kaiser, S., Øvergård, K. T., \& Rosenvinge, J. H. (2014). Comorbidity of personality disorders in mood disorders: A meta-analytic review of 122 studies from 1988 to 2010. Journal of Affective Disorders, 152, 1-11. https://doi.org/10.1016/j.jad.2013.08.023.

Fried, E. I., \& Nesse, R. M. (2015). Depression is not a consistent syndrome: An investigation of unique symptom patterns in the STAR* D study. Journal of Affective Disorders, 172, 96-102. https://doi.org/10.1016/j.jad.2014.10.010.

Fournier, J. C., DeRubeis, R. J., Shelton, R. C., Gallop, R., Amsterdam, J. D., \& Hollon, S. D. (2008). Antidepressant medications v. cognitive therapy in people with depression with or without personality disorder. The British Journal of Psychiatry, 192, 124-129. https://doi.org/10.1192/bjp.bp.107.037234.

Gunderson, J. G. (2014). Handbook of good psychiatric management for borderline personality disorder. Washington, DC: American Psychiatric Association Publishing.

Hamilton, W.K., Aydin, B., \& Mizumoto, A. (2016). MAVIS: Meta analysis via Shiny [Online]. Retrieved December 31, 2020, from http://kylehamilton.net/shiny/MAVIS/.

Hardy, G. E., Barkham, M., Shapiro, D. A., Stiles, W. B., Rees, A., \& Reynolds, S. (1995). Impact of cluster c personality disorders on outcomes of contrasting brief psychotherapies for depression. Journal of Consulting and Clinical Psychology, 63, 997-1004. https://doi.org/10.1037/0022-006X.63.6.997.

Harte, C. B., \& Hawkins, R. C., II. (2016). Impact of personality disorder comorbidity on cognitive-behavioral therapy outcome for mood and anxiety disorders: results from a university training clinic. Research in Psychotherapy: Psychopathology, Process and Outcome, 19, 114-125. https://doi.org/10.4081/ripppo.2016.210. 
Herpertz, S. C., Huprich, S. K., Bohus, M., Chanen, A., Goodman, M., Mehlum, L., et al. (2017). The challenge of transforming the diagnostic system of personality disorders. Journal of Personality Disorders, 31, 577-589. https://doi.org/10.1521/pedi_2017_31_338.

Higgins, J.P.T., Thomas J., Chandler J., Cumpston M., Li T., Page M.J., \& Welch V.A. (eds.). (2019). Cochrane handbook for systematic reviews of interventions version 6.0 (updated July 2019). Cochrane. Retrieved July 17, 2019, from www.training.cochrane. org/handbook.

Higgins, J. P., Thompson, S. G., Deeks, J. J., \& Altman, D. G. (2003). Measuring inconsistency in meta-analyses. British Medical Journal, 327, 557-560. https://doi.org/10.1136/bmj.327.7414.557.

Hirschfeld, R. M. A. (1999). Personality disorders and depression: Comorbidity. Depression and Anxiety, 10, 142-146. https://doi.org/10.1002/(SICI)1520-6394(1999)10:4\%3C142 ::AID-DA2\%3E3.0.CO;2-Q.

Hofmann, S. G., Asnaani, A., Vonk, I. J., Sawyer, A. T., \& Fang, A. (2012). The efficacy of cognitive behavioral therapy: A review of meta-analyses. Cognitive Therapy and Research, 36(5), 427-440. https://doi.org/10.1007/s10608-012-9476-1.

Huprich, S. K. (2019). Personality-driven depression: The case for malignant self-regard (and depressive personalities). Journal of Clinical Psychology, 75, 834-845. https://doi.org/10.1002/ jclp.22760.

Joyce, P. R., McKenzie, J. M., Carter, J. D., Rae, A. M., Luty, S. E., Frampton, C. M., \& Mulder, R. T. (2007). Temperament, character and personality disorders as predictors of response to interpersonal psychotherapy and cognitive-behavioural therapy for depression. The British Journal of Psychiatry, 190, 503-508. https ://doi.org/10.1192/bjp.bp.106.024737.

Kealy, D., \& Ogrodniczuk, J. S. (2010). Marginalization of borderline personality disorder. Journal of Psychiatric Practice, 16, 145-154. https://doi.org/10.1097/01.pra.0000375710.39713.4d.

Kikkert, M. J., Driessen, E., Peen, J., Barber, J. P., Bockting, C., Schalkwijk, F., et al. (2016). The role of avoidant and obsessivecompulsive personality disorder traits in matching patients with major depression to cognitive behavioral and psychodynamic therapy: A replication study. Journal of Affective Disorders, 205, 400-405. https://doi.org/10.1016/j.jad.2016.08.017.

Köhling, J., Ehrenthal, J. C., Levy, K. N., Schauenburg, H., \& Dinger, U. (2015). Quality and severity of depression in borderline personality disorder: asystematic review and meta-analysis. Clinical Psychology Review, 37, 13-25. https://doi.org/10.1016/j. cpr.2015.02.002

Kool, S., Schoevers, R., de Maat, S., Van, R., Molenaar, P., Vink, A., \& Dekker, J. (2005). Efficacy of pharmacotherapy in depressed patients with and without personality disorders: A systematic review and meta-analysis. Journal of Affective Disorders, 88, 269-278. https://doi.org/10.1016/j.jad.2005.05.017.

Kuyken, W., Kurzer, N., DeRubeis, R. J., Beck, A. T., \& Brown, G. K. (2001). Response to cognitive therapy in depression: The role of maladaptive beliefs and personality disorders. Journal of Consulting and Clinical Psychology, 69, 560-566. https://doi. org/10.1037/0022-006X.69.3.560.

Luyten, P., Lowyck, B., \& Blatt, S. J. (2017). Mechanisms of change through the lens of two-polarities models of personality development: State of the art and new directions. Psychoanalytic Inquiry, 37, 179-190. https://doi.org/10.1080/07351690.2017.1285187.

McGlashan, T. H., Grilo, C. M., Skodol, A. E., Gunderson, J. G., Shea, M. T., Morey, L. C., et al. (2000). The collaborative longitudinal personality disorders study: Baseline axis I/II and II/II diagnostic co-occurrence. Acta Psychiatrica Scandinavica, 102(4), 256-264. https://doi.org/10.1034/j.1600-0447.2000.102004256.x.

Moran, P., Romaniuk, H., Coffey, C., Chanen, A., Degenhardt, L., Borschmann, R., \& Patton, G. C. (2016). The influence of personality disorder on the future mental health and social adjustment of young adults: A population-based, longitudinal cohort study. The Lancet Psychiatry, 3, 636-645. https://doi. org/10.1016/S2215-0366(16)30029-3.

Mulder, R. (2002). Personality pathology and treatment outcome in major depression. American Journal of Psychiatry, 159, 359371. https://doi.org/10.1176/appi.ajp.159.3.359.

National Institute for Health and Care Excellence. (2011). Common mental health problems: Identification and pathways to care. London: National Collaborating Centre for Mental Health.

Newton-Howes, G., Johnson, T., \& Tyrer, P. (2006). Personality disorder and the outcome of depression: Systematic review and meta-analysis. The British Journal of Psychiatry, 18, 13-20. https://doi.org/10.1192/bjp.188.1.13.

Newton-Howes, G., Tyrer, P., Johnson, T., Mulder, R., Kool, S., Dekker, J., \& Schoevers, R. (2014). Influence of personality on the outcome of treatment in depression: Systematic review and meta-analysis. Journal of Personality Disorders, 28, 577-593. https://doi.org/10.1521/pedi_2013_27_070.

Orwin, R. G. (1983). A Fail-safe $\mathrm{N}$ for effect size in meta-analysis. Journal of Education Statistics, 8, 157-159.

Paris, J. (2020). Access to psychotherapy for patients with personality disorders. Personality and Mental Health, 14, 246-253. https ://doi.org/10.1002/pmh.1483.

Paris, J. (2013). Stepped care: an alternative to routine extended treatment for patients with borderline personality disorder. Psychiatric Services, 64(10), 1035-1037. https://doi.org/10.1176/ appi.ps.201200451.

Porter, C., Palmier-Claus, J., Branitsky, A., Mansell, W., Warwick, H., \& Varese, F. (2020). Childhood adversity and borderline personality disorder: A meta-analysis. Acta Psychiatrica Scandinavica, 141(1), 6-20. https://doi.org/10.1111/acps.13118.

Rubio-Aparicio, M., Sánchez-Meca, J., López-López, J. A., Botella, J., \& Marín-Martínez, F. (2017). Analysis of categorical moderators in mixed-effects meta-analysis: Consequences of using pooled versus separate estimates of the residual between-studies variances. British Journal of Mathmatical and Statisical Psychology, 70, 439-456. https://doi.org/10.1111/bmsp.12092.

Shea, M. T., Pilkonis, P. A., Beckham, E., Collins, J. F., Elkin, I., Sotsky, S. M., \& Docherty, J. P. (1990). Personality disorders and treatment outcome in the NIMH treatment of depression collaborative research program. American Journal of Psychiatry, 147, 711-718. https://doi.org/10.1176/ajp.147.6.711.

Simonsen, S., Bateman, A., Bohus, M., Dalewijk, H. J., Doering, S., Kaera, A., et al. (2019). European guidelines for personality disorders: Past, present and future. Borderline Personality Disorder and Emotion Dysregulation, 6(1), 9. https://doi. org/10.1186/s40479-019-0106-3.

Snowden, P., \& Kane, E. (2003). Personality disorder: no longer a diagnosis of exclusion. Psychiatric Bulletin, 27(11), 401-403. https://doi.org/10.1192/pb.27.11.401.

Spong, A. J., Clare, I. C. H., Galante, J., Crawford, M. J., \& Jones, P. B. (2020). Brief psychological interventions for borderline personality disorder A systematic review and meta-analysis of randomised controlled trials. Clinical Psychology Review, 83, 101937. https://doi.org/10.1016/j.cpr.2020.101937.

Stalker, K., Ferguson, I., \& Barclay, A. (2005). It is a horrible term for someone': Service user and provider perspectives on 'personality disorder. Disability and Society, 20, 359-373. https:// doi.org/10.1080/09687590500086443.

Sterne, J. A., Savović, J., Page, M. J., Elbers, R. G., Blencowe, N. S., Boutron, I., et al. (2019). RoB 2: A revised tool for assessing risk of bias in randomised trials. BMJ. https://doi.org/10.1136/ bmj.14898.

Tyrer, P., Mitchard, S., Methuen, C., \& Ranger, M. (2003). Treatment rejecting and treatment seeking personality disorders: Type $\mathrm{R}$ 
and Type S. Journal of Personality Disorders, 17(3), 263-268. https://doi.org/10.1521/pedi.17.3.263.22152.

Tyrer, P., Seivewright, N., Ferguson, B., Murphy, S., Darling, C., Brothwell, J., et al. (1990). The Nottingham study of neurotic disorder: Relationship between personality disorder status and symptoms. Psychological Medicine, 20, 423-431. https://doi. org/10.1017/S0033291700017736.

van Bronswijk, S. C., Lemmens, L. H. J. M., Viechtbauer, W., Huibers, M. J. H., Arntz, A., \& Peeters, F. P. M. L. (2018). The impact of personality disorder pathology on the effectiveness of cognitive therapy and interpersonal psychotherapy for major depressive disorder. Journal of Affective Disorders, 225, 530-538. https://doi. org/10.1016/j.jad.2017.08.043.

Van, H. L., \& Kool, M. (2018). What we do, do not, and need to know about comorbid depression and personality disorders. The Lancet Psychiatry, 5(10), 776-778. https://doi.org/10.1016/S2215 -0366(18)30260-8.

van den Hout, M., Brouwers, C., \& Oomen, J. (2006). Clinically diagnosed axis II co-morbidity and the short term outcome of CBT for axis I disorders. Clinical Psychology and Psychotherapy, 13, 56-63. https://doi.org/10.1002/cpp.477.
Winsper, C., Bilgin, A., Thompson, A., Marwaha, S., Chanen, A. M., Singh, S. P., et al. (2019). The prevalence of personality disorders in the community: A global systematic review and metaanalysis. The British Journal of Psychiatry, 12, 1-10. https://doi. org/10.1192/bjp.2019.166.

Wongpakaran, N., Wongpakaran, T., Boonyanaruthee, V., Pinyopornpanish, M., \& Intaprasert, S. (2015). Comorbid personality disorders among patients with depression. Neuropsychiatric Disease and Treatment, 11, 1091-1096.

Zanarini, M. C., Hörz-Sagstetter, S., Temes, C. M., Frankenburg, F. R., Hein, K. E., Reich, D. B., \& Fitzmaurice, G. M. (2019). The 24-year course of major depression in patients with borderline personality disorder and personality-disordered comparison subjects. Journal of Affective Disorders, 258, 109-114. https://doi. org/10.1016/j.jad.2019.08.005.

Publisher's Note Springer Nature remains neutral with regard to jurisdictional claims in published maps and institutional affiliations. 